\title{
sistemas de control automático en pavimentadoras acabadoras
}

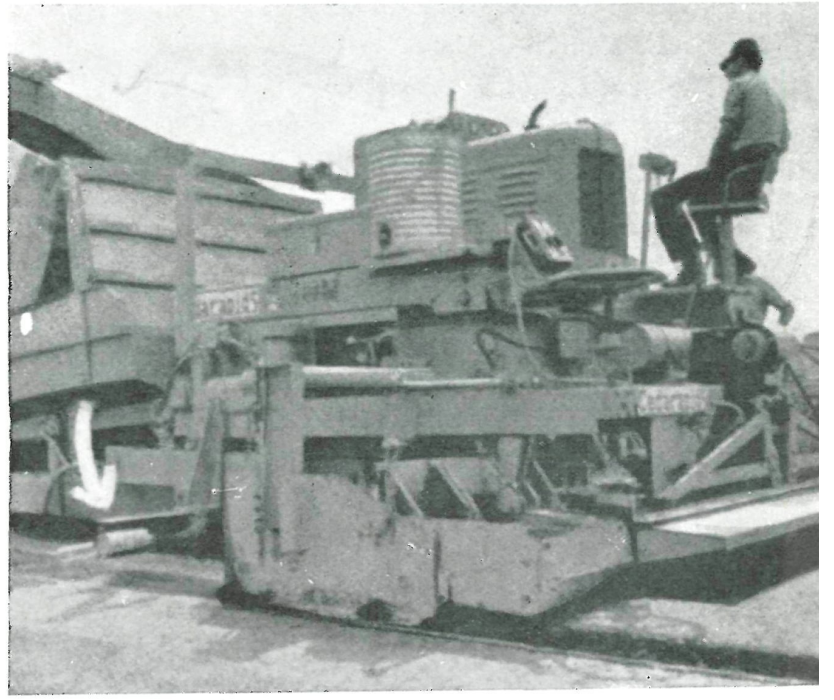

Los sistemas de control automático tienden a lograr la fijación de la posición de la placa acabadora, de forma que, independientemente de las irregularidades del suelo y alteraciones producidas por las ruedas y orugas durante el movimiento de máquina, se consiga una superficie plana uniforme y del espesor y pendiente deseados.

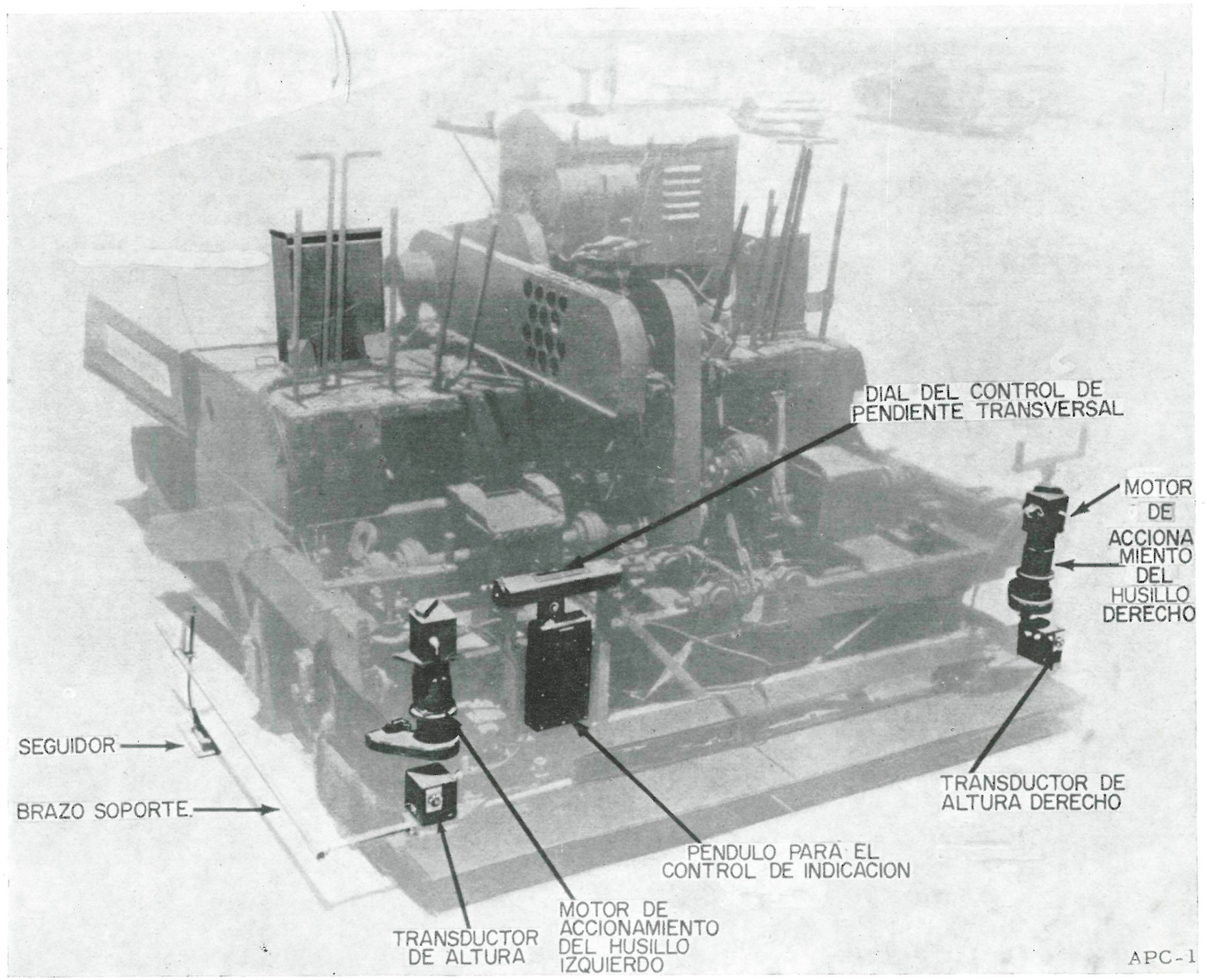




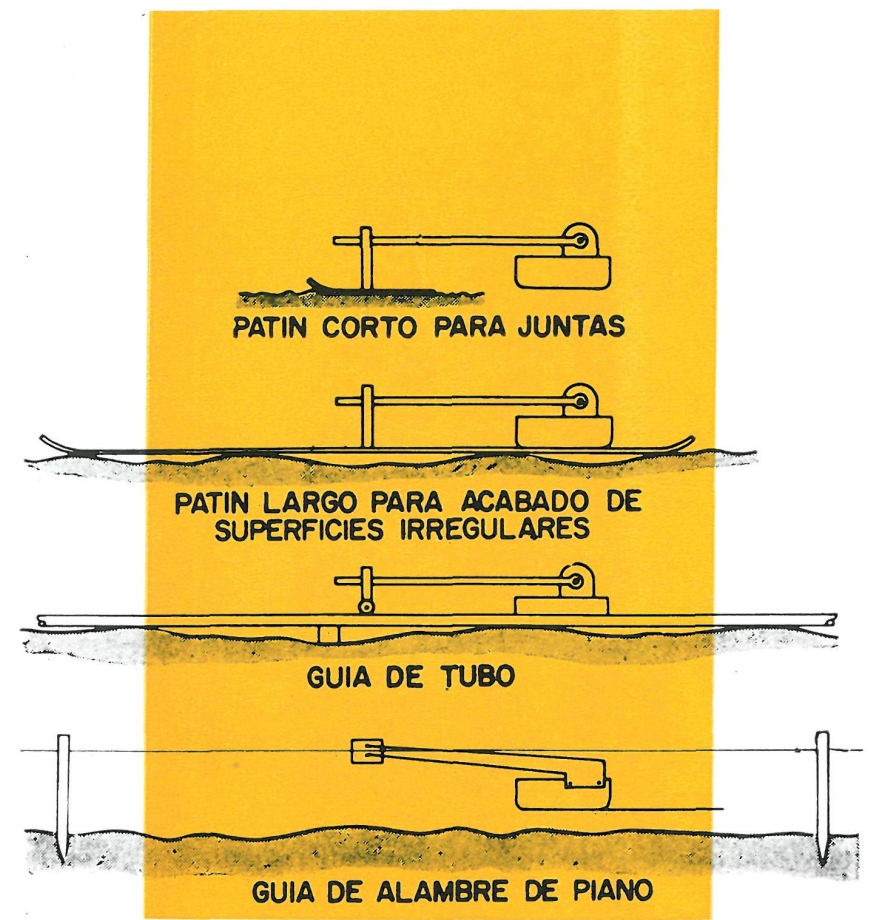

Para ello es preciso disponer de una superficie o línea de referencia previamente establecida; generalmente, un bordillo, regla metálica, alambre de piano estirado o el borde de un pavimento realizado anteriormente.

Las ventajas que se pretenden lograr con los sistemas automáticos de control son:

Mayor uniformidad y homogeneidad de las superficies producidas.

Juntas más perfectas.

Mayores velocidades de trabajo.

Disminución del costo al emplear menos mano de obra y obtener mayores velocidades de trabajo.

Permitir la realización de capas de características fijas y uniformes sobre suelos irregulares, ya que los movimientos verticales de la placa acabadora son independientes del movimiento de los puntos de apoyo de la máquina.

Entre otras empresas, la Iowa Mfg Co., en conjunción con la Minneapolis Honeywell y la Preco Incorporated, están realizando un amplio programa de investigación y desarrollo de los sistemas automáticos de control.

Dos prototipos de este nuevo sistema han sido montados por la Preco Inc. en pavimentadoras Barber Greene, S. A., 40, con el fin de lograr la debida experiencia, relativa al funcionamiento mecánico del sistema de control.

Como la técnica de empleo sufrirá modificaciones en consonancia con las nuevas características de trabajo, la segunda parte del programa consistirá en situar un número limitado de pavimentadoras dotadas de control automático, en la zona Sur de California, con objeto de obtener una amplia experiencia que permita la deducción de normas de emplec adecuadas.

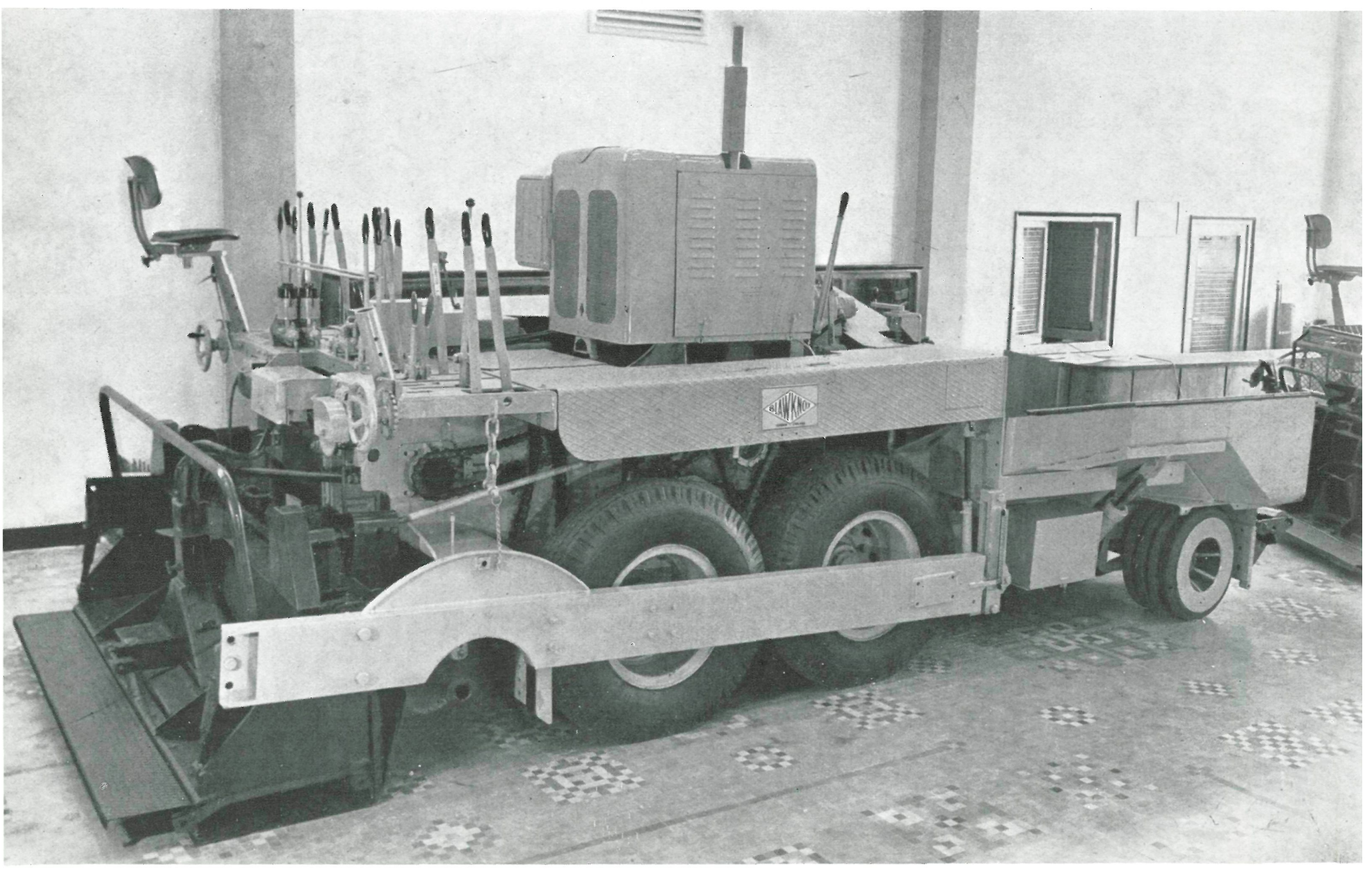




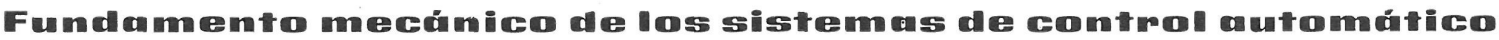

De acuerdo con el espesor y perfil transversal deseados, la altura de la placa acabadora y su ángulo de ataque son controlados por un palpador o seguidor que se desplaza por la superficie o línea de referencia. Los movimientos del seguidor son transmitidos, por su brazo soporte, a un transductor, en el que se miden los movimientos angulares y se convierten en señales eléctricas, las cuales, recibidas en la caja de control, son transformadas en impulsos que actúan los servomotores de accionamiento de los husillos de elevación.

La posición inicial de la placa acabadora se fija manualmente por el operador, sin que haya necesidad posterior de ajustes manuales de posición, los que se verifican automáticamente por el sistema de control.

Normalmente la máquina sólo está provista de un seguidor en uno de sus costados, el cual puede cambiarse al otro lado mediante una sencilla operación de pocos minutos de duración. Se pueden usar seguidores en ambos lados de la máquina simultáneamente, como sucede en los casos en los que la franja que se esté realizando se halle situada en otras dos anteriormente establecidas.

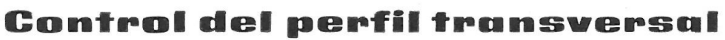

El elemento básico es un péndulo montado en un marco rígidamente unido a los extremos de la máquina. El operador fija en la escala correspondiente el perfil transversal deseado, con independencia de la altura de franqueo de la placa acabadora.

Las variaciones de pendiente transversal sufridas por la placa acabadora durante el movimiento de la máquina son acusadas y medidas por el péndulo; las señales eléctricas correspondientes enviadas a la caja de control, corrigen la posición de la placa mediante el accionamiento del servomotor del husillo correspondiente.

\section{Caja de comtrol}

Los dispositivos electrónicos están concentrados en la caja ce control, pintada con pintura reflexiva al calor y montada de forma que no sufra las vibraciones de la máquina.

La caja de control contiene tres sistemas de amplificadores, que reciben las señales de los transductores y las convierten en órdenes para la activación de los servomotores de accionamiento de los husillos.

El control puede operar automática o semiautomáticamente, pudiendo cambiarse simultáneamente el ángulo de ataque y la altura de la placa.

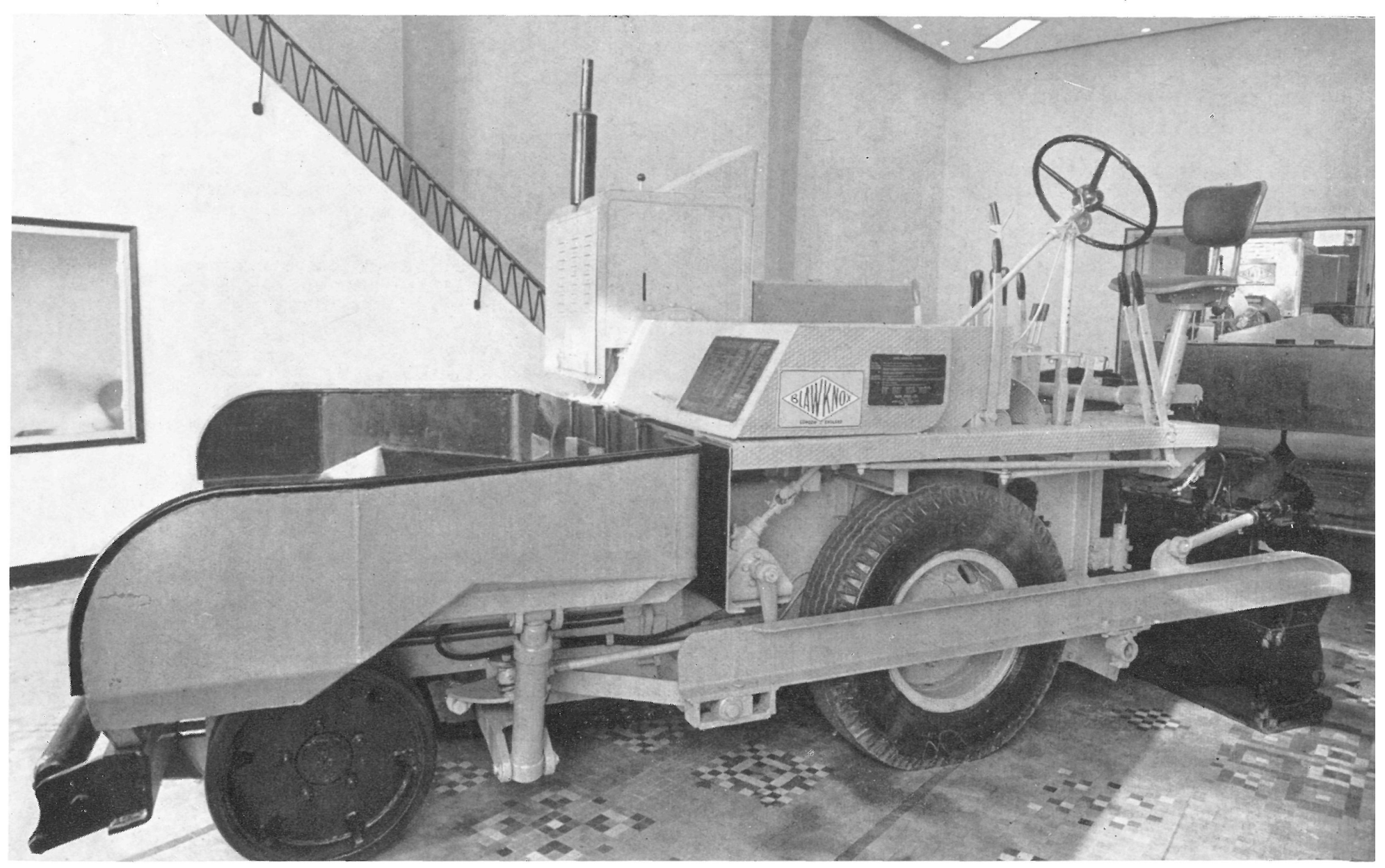




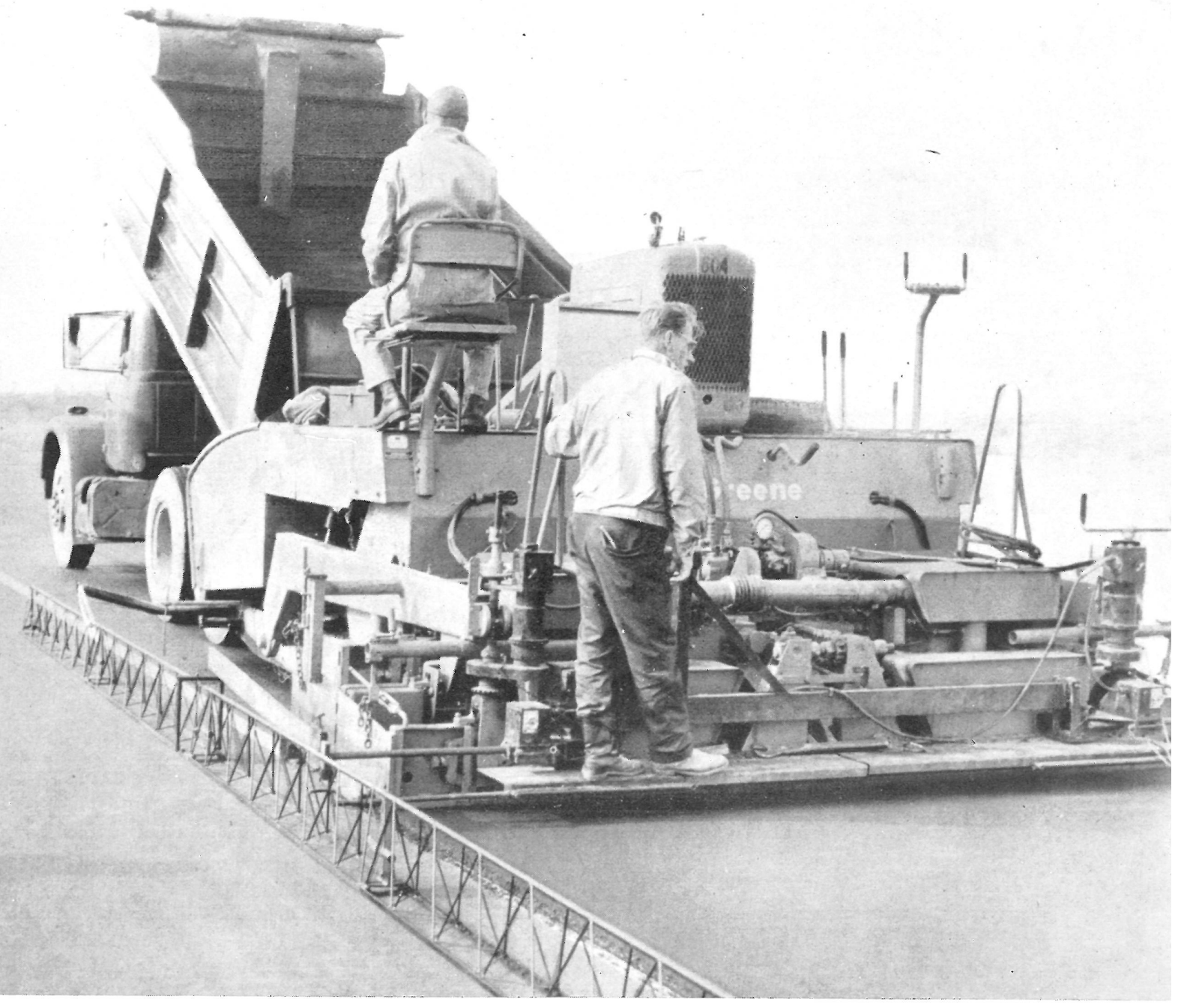

\section{Seguidor o prolpactor}

De acuerdo con el trabajo que se esté realizando, se fijan diversos dispositivos seguidores en el brazo soporte

En curvas, para la realización de juntas, o cuando la superficie de referencia sea suficientemente plana, se usa un patín de pequeña longitud. Para trabajos de acabado, o cuando la superficie de referencia presente irregularidades, se utiliza un patín largo de hasta 9 metros de longitud, el cual, al deslizarse sobre las crestas, proporciona la capa de espesor mínimo necesario.

Además de la guía de referencia de alambre de piano estirado, la Preco Ing. ha experimentado, con buenos resultados para trabajos rápidos, la utilización de una guía, que consiste en un tubo de aluminio, de 3 pulgadas de diámetro, pudiendo ser el palpador un rodillo ancho giratorio, o un patín corriente.

\section{El Electromatic Screed Controuler}

El sistema de control automático experimentado por la Iowa Manufacturing Company difiere poco del expuesto anteriormente.

El horizonte artificial se consigue, en vez de con el péndulo, con un cilindro de acero flotante en un baño de aceite. El conjunto está compuesto con un panel de mando, un péndulo para horizonte artificial, un palpador de superficie, una caja de control y dos servomotores para el accionamiento de la placa acabadora.

Las señales pueden ser enviadas a la caja de control desde el panel de mando, el sensor o el péndulo, siendo transformada en impulsos que activan los servomotores, los cuales accionan el movimiento de tijera de los brazos de la acabadora Cedar Rapids, sobre la que se ha experimentado este sistema, modificando la posición de la placa acabadora.

Este sistema de control ha sido experimentado ampliamente en Iowa por la Highway Surfacers Incorporated.

\section{CHINCHILLA}

Agradeciendo sinceramente la información facilitada por la Preco Incorporated, de los Angeles, y la Hispano Toledana, S. L., de Madrid. 